\title{
ANTI-CANCER DRUG DOXORUBICIN INDUCED CARDIOTOXICITY: UNDERSTANDING THE MECHANISMS INVOLVED IN ROS GENERATION RESULTING IN MITOCHONDRIAL DYSFUNCTION
}

\author{
Shishir Upadhayay ${ }^{1}$, Nidhi Sharma ${ }^{2}$ Anil K. Mantha ${ }^{1}$ and Monisha Dhiman ${ }^{3, *}$ \\ ${ }^{1}$ Department of Zoology, School of Basic and Applied Sciences, Central University of Punjab, \\ Bathinda, 151001, Punjab, India. \\ ${ }^{2}$ Department of Human Genetics and Molecular Medicine, Central University of Punjab, \\ Bathinda, 151001, Punjab, India \\ ${ }^{3}$ Department of Microbiology, School of Basic and Applied Sciences, Central University of \\ Punjab, Bathinda, 151001, Punjab, India \\ *E-mail: monisha.dhiman@cup.edu.in
}

\begin{abstract}
Doxorubicin (DOX), despite being an effective anti-cancer drug has offsite targets that affect the vital organs such as heart, brain and kidney. DOX-induced cardiotoxicity is reported as a multi-factorial process that interferes with mitochondrial bioenergetics. These responses increase the threshold of oxidant-mediated injury and redox-mediated apoptosis in the cardiomyocytes. Oxidative stress particularly mitochondrial dysfunction in cardiomyocytes associated with cardiovascular diseases. In the present study we examined the effect of DOX on H9c2 cardiomyocyte where cells were treated with $5 \mu \mathrm{M}$ DOX. To rule out the source of reactive oxygen species (ROS) during DOX-induced toxicity, the DOX-treated cardiomyocytes were incubated with $100 \mu \mathrm{M}$ diphenyleneiodonium (DPI), $50 \mu \mathrm{M}$ salicyl hydroxamic acid (SHX), $20 \mu \mathrm{M}$ Carbonyl cyanide-4-(trifluoromethoxy) phenylhydrazone (FCCP), which are specific inhibitors of NADPH oxidase (NOX), Myeloperoxidase (MPO), and mitochondrial oxidative phosphorylation respectively and $10 \mu \mathrm{M} \mathrm{N}$-acetyl cysteine (NAC, free radical scavenger) was also used to perceive the role of ROS. $\mathrm{H}_{2} \mathrm{O}_{2}(100 \mu \mathrm{M})$ treated $\mathrm{H} 9 \mathrm{c} 2$ cardiomyocytes were used as positive controls. The cell viability, reactive oxygen species (ROS) level and oxidative stress were determined using MTT assay, NBT assay/Flow-cytometry and Western blotting based assays. The effect of DOX on mitochondria was evaluated using Amplex Red assay; fluorescent probes such as MitoSOX and MitoTracker were used to examine the DOX-induced ROS production from the mitochondrial matrix. The mitochondrial membrane potential was evaluated using JC-1 dye. Western blotting was performed for cytochrome c release and apoptosis was examined with Annexin V-FITC assay. DOX was found to reduce cell viability, increase ROS level followed by enhanced oxidative stress in the form of protein carbonyls. DOX also showed a reduction in the mitochondrial membrane potential and allowed the release of cytochrome $\mathrm{c}$ which further leads to apoptosis and cell death. Further to rule out the pathway/mechanism(s) of DOX-mediated cardiac pathologies, the treatment with inhibitors of the classical ROS sources such as NADPH oxidase, Myeloperoxidase, mitochondria and general ROS scavenger (NAC) suggested that ROS via NOX and MPO during DOX-induced toxicity plays a crucial role in cardiomyocytes. The mitochondrial integrity was conserved when the cells were treated with NOX and MPO inhibitors, the cytochrome C release and apoptosis reduced in presence of these inhibitors. Taken together, these results demonstrate that DOX leads to ROS production and oxidative stress in cardiomyocytes which ultimately affects the mitochondrial integrity and functions, most importantly the ROS released via NOX and MPO is critical during DOX-induced cardiotoxicity.
\end{abstract}

Keywords: Anthracyclines, Cardiotoxicity, Chemotherapy, Mitochondrial Dysfunction, Reactive Oxygen Species (ROS).

(C) RASĀYAN. All rights reserved

\section{INTRODUCTION}

Cardiovascular diseases (CVDs) are the leading cause of mortality worldwide. There is a remarkable relationship between reactive oxygen species (ROS), oxidative stress and CVDs. ROS, as well as reactive Rasayan J. Chem., 13(2), 1042-1053(2020) http://dx.doi.org/10.31788/RJC.2020.1325603

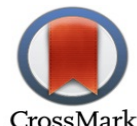


nitrogen species (RNS), as a product of normal cellular metabolism, play a dual role since they can be either harmful or beneficial to living systems. ${ }^{1}$ ROS/RNS are either generated through endogenous sources such as by-products of the mitochondrial respiratory chain, activation of various enzymes such as NADPH oxidase (NOX), xanthine oxidase, myeloperoxidase or through exogenous sources. Oxidative stress is the result of an imbalance between the level of free radical production and antioxidant level that has been implicated in several human diseases. Oxidative stress can damage cellular lipids, proteins, or DNA thus inhibits their normal function. Cardiac cells are susceptible to free radical damage because of their high oxidative metabolism and relatively poor antioxidant defense system. ${ }^{2}$ Mitochondria being the chief source of energy, the heart function majorly depends on the highly efficient mitochondria. The electron transport chain of mitochondria is the major contributor of free radical generation, apoptosis and energy metabolism, thus any defect in the normal functioning of mitochondria leads to the development of CVDs and related complications. ${ }^{3}$

Cancer is the second most fatal disease that is a bane to human life all over the world. In cancer, cells divide and grow overwhelmingly, forming malignant tumors, and metastasizes the other tissues of the body. ${ }^{4}$ Various chemotherapeutic drugs employed to cure cancer have increased the survival rate of the cancer patients but these drugs come along with many side effects. These drugs increase the level of ROS in mitochondria that ultimately leads to mitochondrial dysfunction which ultimately affects the function of heart due to cardiotoxicity. Cardiotoxicity is a big concern in cancer therapy and the long-term survivors include pediatrics cancer patients and elderly patients with pre-existing cardiovascular comorbidities are at a greater risk. The use of combination immuno-chemotherapy with overlapping toxicities (e.g. trastuzumab, taxanes, and anthracyclines) is another matter of concern. ${ }^{5}$

Anthracycline is the most famous class of chemotherapeutic drugs and doxorubicin (DOX) belonging to this family is one of the most effective anticancer drugs and has been widely used in the treatment of several adult and pediatric cancers, leukemia, lymphomas and breast cancer. DOX act by intercalating into the nuclear DNA, inhibiting the function of topoisomerase II, generation of ROS and inducing apoptosis in the cancerous cells. A major limitation of DOX usage is life-threatening cumulative dosedependent cardiac toxicity. ${ }^{6}$ DOX shows its cardiotoxic effect by increasing the level of iron and ROS that ultimately affects the cardiovascular system. ${ }^{7}$ DOX-associated cardiotoxicity can be categorized as immediate pericarditis-myocarditis syndrome, early-onset chronic progressive form and late-onset chronic progressive form. The most troublesome is the late chronic cardiotoxicity, which develops years after the treatment and is typically characterized by myofibril loss and degeneration ventricular cardiomyocytes, ultimately resulting in dilated cardiomyopathy and heart failure.

The objective of the present study is to detect the cardiotoxicity induced by doxorubicin and to demonstrate the associated mechanism. The H9c2 (rat cardiomyocytes) cell line was used to detect the DOX-induced cell death, ROS levels, oxidative stress, mitochondrial damage and apoptosis. The results suggest that DOX treatment enhances the ROS levels and oxidative stress which ultimately affects the mitochondrial functioning. Subsequently, higher levels of cytochrome c release suggest the leaky membrane and cells being in the late apoptosis stage in DOX treated cells. Further to rule out the pathway/mechanism(s) of DOX-mediated cardiac pathologies the treatment with inhibitors of the classical ROS sources such as NADPH oxidase, Myeloperoxidase, mitochondria and general ROS scavenger (NAC) suggested that ROS via NOX and MPO during DOX-induced toxicity plays a crucial role in cardiomyocytes. This study will provide directions for the development of adjunct therapies to control oxidative disarray occurring during chemotherapy resulting in heart failure and related complications.

\section{EXPERIMENTAL}

\section{Culturing and Treatment of $\mathrm{H} 9 \mathrm{c} 2$ Cells}

H9c2 (2-1) rat cardiomyocytes were procured from NCCS, Pune. The cells were grown in DMEM supplemented with $10 \%$ FBS, antibiotics $(100 \mathrm{U} / \mathrm{ml}$ of Penicillin, and $100 \mu \mathrm{g} / \mathrm{ml}$ of Streptomycin), 1.5 $\mathrm{g} / \mathrm{L}$ sodium bicarbonate and incubated at $37^{\circ} \mathrm{C}, 5 \% \mathrm{CO}_{2}$ humidified atmosphere and passaged every 2-3 days after trypsinization with trypsin/EDTA. The cardiomyocytes were treated for $24 \mathrm{hr}$ with various concentrations of DOX to determine the most effective concentration which was toxic to cells. To rule out the source of ROS, DOX-treated cardiomyocytes were incubated along with $100 \mu \mathrm{M}$ 
diphenyleneiodonium (DPI), $50 \mu \mathrm{M}$ salicyl hydroxamic acid (SHX), $20 \mu \mathrm{M}$ Carbonyl cyanide-4(trifluoromethoxy) phenylhydrazone (FCCP), specific inhibitors of NADPH oxidase (NOX), Myeloperoxidase (MPO), and mitochondrial oxidative phosphorylation respectively and $10 \mu \mathrm{M}$ free radical scavenger $\mathrm{N}$-acetyl cysteine (NAC). Simultaneously, $100 \mu \mathrm{M} \mathrm{H}_{2} \mathrm{O}_{2}$ treated $\mathrm{H} 9 \mathrm{c} 2$ cardiomyocytes were used as a positive control.

\section{MTT Assay for Cytotoxicity}

Cardiomyocytes $\left(1 \times 10^{4}\right.$ per well) were cultured in a 96 well plate and treated with various concentrations of DOX $(1 \mu \mathrm{M}, 3 \mu \mathrm{M}, 5 \mu \mathrm{M}$ and $7 \mu \mathrm{M})$ and incubated for $24 \mathrm{hr}$. The cells were incubated with MTT solution $(5 \mathrm{mg} / \mathrm{ml})$ for $3 \mathrm{hr}$ until purple colored crystals were visible. The formazan crystals were completely solubilized in DMSO and absorbance was recorded at $570 \mathrm{~nm}$ in Synergy H1 microplate reader. Each concentration of DOX was tested in replicates. Cytotoxic effect of DOX was expressed as relative viability (percent control) concerning negative control where the percentage of cell survival in negative control was taken as $100 .^{8}$

\section{Giemsa Staining for Cell Morphology}

H9c2 cells $\left(1 \times 10^{5}\right)$ we re cultured and treated with $5 \mu \mathrm{M}$ concentration of DOX in Lab-Tek II chamber slides and incubated at $37^{\circ} \mathrm{C} / 5 \% \mathrm{CO}_{2}$ for $24 \mathrm{hr}$. Cells were fixed in absolute methanol $(200 \mu \mathrm{l})$ followed by staining with Wright-Giemsa stain for $20 \mathrm{~min}$. Images were visualized under the light microscope (Cx25 OLYMPUS) at 100X. ${ }^{9}$

\section{Detection of ROS Generation NBT Assay}

Cardiomyocytes $\left(1 \mathrm{X} 10^{5}\right.$ per well) were cultured and treated with various concentrations of DOX (1-7 $\mu \mathrm{M})$ and incubated for $24 \mathrm{hr}$. In some experiments the DOX $(5 \mu \mathrm{M})$ treated cells were also treated with various ROS scavengers, $100 \mu \mathrm{M}$ DPI, $50 \mu \mathrm{M}$ SHX, $20 \mu \mathrm{M}$ FCCP and $10 \mu \mathrm{M}$ NAC. The cells were then incubated with $0.1 \% \mathrm{NBT}(1 \mathrm{mg} / \mathrm{ml})$ at $37^{\circ} \mathrm{C} / 5 \% \mathrm{CO}_{2}$ for $1 \mathrm{hr}$ in dark. NBT was removed and cells were washed with 1X PBS and then with $70 \%$ methanol followed by snap cooling with $100 \%$ chilled methanol. $2 \mathrm{M} \mathrm{KOH}$ and DMSO were used to dissolve the crystals. Absorbance was measured at $620 \mathrm{~nm}$ in Synergy H1 microplate reader and percent ROS was calculated. ${ }^{9}$ For microscopic NBT Assay H9c2 cells $\left(1 \mathrm{X} 10^{5}\right)$ were cultured and treated with DOX $(5 \mu \mathrm{M})$ in Lab- Tek II chamber slides. The cells were incubated with $0.1 \%$ NBT $(1 \mathrm{mg} / \mathrm{ml})$ washed then stained with safranin stain for $15-20 \mathrm{~min}$ followed by washing and mounting with DPX. Images were visualized under the light microscope (Cx25 OLYMPUS) at $100 \mathrm{X}^{10}$

\section{Flow-Cytometric Analysis of ROS/RNS in H9c2 Cardiomyocytes}

DHE and DAF-FM diacetate are cell-permeable fluorescent probes used for the quantification of free radical and intracellular nitric oxide (NO) respectively. DAF-FM probe diffuses through cellular membranes and is converted into a fluorescent product in the presence of intracellular esterases, which is quantified at excitation/emission maxima of $495 / 515 \mathrm{~nm} .{ }^{11} 1 \times 10^{4}$ cells were seeded in 96-well plates and treated with DOX and incubated at $5 \% \mathrm{CO}_{2}$ and $95 \% \mathrm{O}_{2}$. After washing with $1 \mathrm{X}$ PBS, DAF-FM/DHE was added to each well followed by incubation for $60 \mathrm{~min}$ at room temperature. The DAF-FM was washed and replaced with 1X PBS and incubated for $15 \mathrm{~min}$. Fluorescence was measured by using BD Accuri C6.

\section{Preparation of Total Cell Extracts}

The DOX $(5 \mu \mathrm{M})$ treated H9c2 cells cultured with or without ROS scavengers were trypsinized and centrifuged at $2000 \mathrm{rpm}$ for $10 \mathrm{~min}$. The pellet was lysed in ice-cold lysis buffer [150 mM NaCl, $20 \mathrm{mM}$ Tris-HCl, $1 \mathrm{mM}$ EDTA, $0.1 \% \mathrm{NP}-40$, protease inhibitor (1X)] for $30 \mathrm{~min}$ followed by centrifugation at $20,000 \mathrm{~g}$ for $15 \mathrm{~min}$ at $4^{0} \mathrm{C}$. The supernatant was stored at $-20^{\circ} \mathrm{C}$ (Rhein et al., 2010). The amount of protein in the total cell extract of different treatment groups was determined by the Bradford method using Bovine Serum Albumin (BSA) as standard. 


\section{Determination of Oxidative Stress using Protein Carbonyl Assay}

The cell lysate was derivatized with $20 \mathrm{mM} \mathrm{DNPH}$, the derivatized and non-derivatized samples were separated on SDS-PAGE and were transferred on to nitrocellulose (NC) membrane using Amersham Bioscience's semidry apparatus. The membrane was then blocked with $1 \%$ bovine serum albumin (BSA) and incubated with primary antibodies directed against the DNPH monoclonal (1:1000). The membrane was washed and further incubated with horseradish peroxidase (HRP) conjugated anti-rabbit secondary antibody (1:5000) Enhanced chemiluminescence reagent was used as a substrate to detect the signal followed by imaging and densitometry analysis using Bio-Rad ChemiDoc ${ }^{\mathrm{TM}} \mathrm{MP}$ imaging system. ${ }^{8}$

\section{Measurement of $\mathrm{H}_{2} \mathrm{O}_{2}$ Production by Amplex Red Assay in Mitochondria}

Mitochondria from the treated and untreated cells were isolated by differential centrifugation as described previously ${ }^{12-13}$ and $\mathrm{H}_{2} \mathrm{O}_{2}$ generation was monitored using $100 \mu \mathrm{M}$ 10-acetyl-3, 7dihydroxyphenoxazine (Amplex red) and $0.3 \mathrm{U} / \mathrm{ml} \mathrm{HRP}$. Briefly, the mitochondrial lysate was incubated with Amplex red and HRP in black bottom 96 well plate for $30 \mathrm{~min}$ at room temperature. HRP-catalyzed ample red oxidation by $\mathrm{H}_{2} \mathrm{O}_{2}$ results in the formation of fluorescent resorufin which was monitored at $\mathrm{Ex}_{560 \mathrm{~nm}} / \mathrm{Em}_{590 \mathrm{~nm}}$ using Synergy $\mathrm{H} 1$ microplate reader. A standard curve with $\mathrm{H}_{2} \mathrm{O}_{2}$ (range $50 \mathrm{nM}-5 \mu \mathrm{M}$ ) was prepared for final calculation. ${ }^{14}$

\section{Measurement of Mitochondrial Matrix-specific ROS using Confocal Microscope}

H9c2 cardiomyocytes were cultured a n d treated with DOX along with or without ROS scavengers (described above) and incubated for $48 \mathrm{hr}$. After $48 \mathrm{hr}$ cells were washed with PBS and incubated with $200 \mathrm{nM}$ MitoTracker red (mitochondria-specific, $\mathrm{Ex}_{579 \mathrm{~nm}} / \mathrm{Em}_{599 \mathrm{~nm}}$ ), $5 \mu \mathrm{M}$ MitoSOX red (specifically detects mitochondrial matrix ROS, $\mathrm{Ex}_{510 \mathrm{~nm}} / \mathrm{Em}_{580 \mathrm{~nm}}$ ) for $30 \mathrm{~min}$ and then washed thrice with PBS (Gupta et al., 2009). Subsequently, the cells were analyzed using an Olympus FV-1200 Laser Scanning Confocal Microscope. ${ }^{15}$

\section{Assessment of Mitochondrial Transmembrane Potential ( $\Delta \psi \mathrm{m})$}

Cardiomyocytes cultured in 96 well plates were treated with DOX along with or without inhibitors for 48 hr. After $48 \mathrm{hr}$, cells were washed with PBS and incubated with $(10 \mu \mathrm{M}) \mathrm{JC}-1$ for $30 \mathrm{~min}$. Then cells were washed with PBS and fluorescence was measured using Synergy H1 microplate reader (green monomers with $\mathrm{Ex}_{485 \mathrm{~nm}} / \mathrm{Em}_{535 \mathrm{~nm}}$ and red J-aggregates with $\mathrm{Ex}_{560 \mathrm{~nm}} / \mathrm{Em}_{595 \mathrm{~nm}}$, a sensitive marker of $\left.\Delta \psi \mathrm{m}\right){ }^{16}$

\section{Detection of Mitochondrial Membrane Damage via Cytochrome $\mathbf{C}$ release}

Total cell lysates were prepared as described above and were separated on 12\% SDS/PAGE and then transferred on to nitrocellulose (NC) membrane using Amersham Bioscience's semidry apparatus. The membrane was then blocked with 5\% non-fat dry milk (NFDM) and incubated overnight at $4{ }^{\circ} \mathrm{C}$ with mouse monoclonal primary antibody of cytochrome-c (1:1000). After washing the membrane was incubated with an HRP-conjugated anti-mouse secondary antibody (1:5000). ECL reagent was used as a chemiluminescent substrate to detect the signal followed by imaging and data analysis using the Bio-Rad ChemiDoc ${ }^{\mathrm{TM}}$ MP imaging system. ${ }^{17}$

\section{Detection of Intrinsic Apoptosis: Annexin V-FITC Assay}

For apoptosis detection, fresh cardiomyocytes, with control and positive control groups, were treated with DOX along with or without ROS inhibitors (as described in previous sections) for $24 \mathrm{hr}$. After washing cells were resuspended in binding buffer containing propidium iodide (PI) and Annexin $\mathrm{V}$ conjugated to FITC (1:500 dilution) for $30 \mathrm{~min}$. then washed and fixed with $2 \%$ formaldehyde ${ }^{18}$ and visualized under Olympus FV-1200 Laser Scanning Confocal Microscope using $\mathrm{Ex}_{488 \mathrm{~nm}} / \mathrm{Em}_{530 \mathrm{~nm}}$ for Annexin V and $\mathrm{Ex}_{535 \mathrm{~nm}} / \mathrm{Em}_{617 \mathrm{~nm}}$ for PI. ${ }^{18}$

\section{Statistical Analysis}

The experiments were done in triplicates and assays were repeated at least three times. Data are presented as mean \pm S.D for control as well as experimental samples. For all statistical comparison, Student's t-test was used with the $\mathrm{p}$-value $<0.05$ and $<0.005$ was considered statistically significant. 
RASĀYAN J. Chem.

Vol. 13 | No. 2 |1042 - 1053| April - June | 2020

\section{RESULTS AND DISCUSSION}

In the present study, we examined the role of DOX-induced oxidative stress on the H9c2 cardiac cell line. Our major focus was to see its effects on cardiac mitochondria as the cardiac system requires more efficient mitochondria for the continuous beating of the heart. To counteract the effects of ROS produced by DOX we used ROS scavengers DPI, SHX, FCCP and NAC in our experiments. We used these ROS scavengers along with DOX treatment to elucidate that up to which extent they reduce the level of ROS produced by DOX via inhibiting or not inhibiting particular enzyme activity.

H9c2 Cells treated with 1, 3, 5 and $7 \mu \mathrm{M}$ DOX concentration for $24 \mathrm{hr}$ showed $36 \%, 47 \%, 50 \%$ and $53 \%$ decrease in cell viability respectively as compared to untreated control cells which indicate that DOX induces cell injury and hence reduces the cell viability (Fig.-1a). Our main objective was to examine the role of ROS during DOX-induced toxicity so first, we confirmed the ROS production using nitroblue tetrazolium chloride (NBT) assay. The ROS level was increased by $35 \%, 20 \%, 90 \%$ and $75 \%$ in cardiomyocytes treated with $1 \mu \mathrm{M}, 3 \mu \mathrm{M}, 5 \mu \mathrm{M}$ and $7 \mu \mathrm{M}$ concentrations respectively, as compared to the untreated control cells (Fig.-1b).

Cells when treated with $5 \mu \mathrm{M}$ DOX showed a significant change in their morphology as compared to untreated control cells when stained with Giemsa. Normal cells were spindle-shaped with clear structure while DOX-treated cells lost their normal morphology and were round in shape as well as there was a change in the nuclear morphology (Fig.-2a). The reduction of NBT in presence of ROS leads to the formation of purple-colored formazan crystals in DOX-treated cells, the marked increase in purple crystals (marked with arrows) indicated the production of superoxide anion in DOX-treated cardiomyocytes (Fig.-2b).
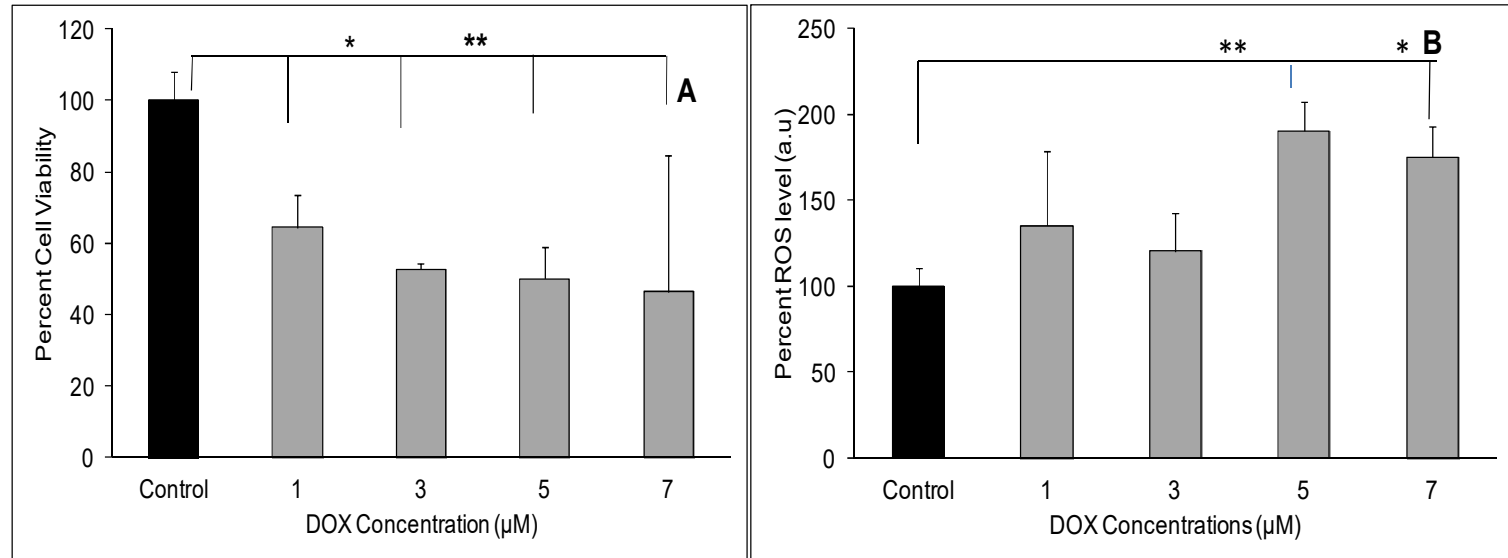

Fig.-1: (A). The Percent Cell Survival after DOX Treatment: The percent viability of H9c2 cells treated with increasing concentration of DOX $(1 \mu \mathrm{M}, 3 \mu \mathrm{M}, 5 \mu \mathrm{M}$ and $7 \mu \mathrm{M})$ for $24 \mathrm{hr}$. The results are expressed as percent cell viability. (B). ROS Production in the DOX treated H9c2 Cells: The percent ROS level in H9c2 cells treated with DOX concentration $(1 \mu \mathrm{M}, 3 \mu \mathrm{M}, 5 \mu \mathrm{M}$ and $7 \mu \mathrm{M})$ for $24 \mathrm{hr}$. The results were expressed as percent ROS level in arbitrary units [a.u]. Student $t$ - test was performed to evaluate the significance of the results, the data was considered as statistically significant at ${ }^{*} \mathrm{p} \leq 0.05$ and ${ }^{* *} \mathrm{p} \leq 0.005$ when DOX-treated cells were compared with untreated controls. The results are presented as mean \pm standard deviation $(n=3)$.

Flow-cytometry based study was performed to assess the ROS level using DHE and RNS level using DAF-FM fluorescent probes. The RNS levels in DOX treated cells showed an increase of 7 fold (Fig.-3a) whereas the ROS was increased by three folds when compared with untreated control (Fig.-3b). The sources of these ROS and RNS might be mitochondria, rapidly degradation in antioxidant activity of superoxide dismutase (SOD)-2 and glutathione or sequester $\mathrm{O}_{2}{ }^{-19}$, or elevating the functioning of ROS generating NADPH oxidase (NOX) ${ }^{20}$, Myeloperoxidase (MPO). ${ }^{21}$

Hydrazone formed after the reaction between DNPH and protein carbonyls was detected by Western blotting. A significantly high amount of protein carbonyl content was observed in DOX-treated cell lysates as compared to control cell lysates in derivatized samples (Fig.-3c). Densitometric analysis of 
RASĀYAN J. Chem.

Vol. 13 | No. 2 |1042 - 1053| April - June | 2020

Western blot showed an increase in carbonyl content in DOX-treated cells, indicating more protein oxidation as compared to the untreated control cells (Fig.-3d). Protein carbonylation is an irreversible phenomena due to DOX-induced oxidative stress is also observed in a spontaneously hypertensive rat model. $^{22}$

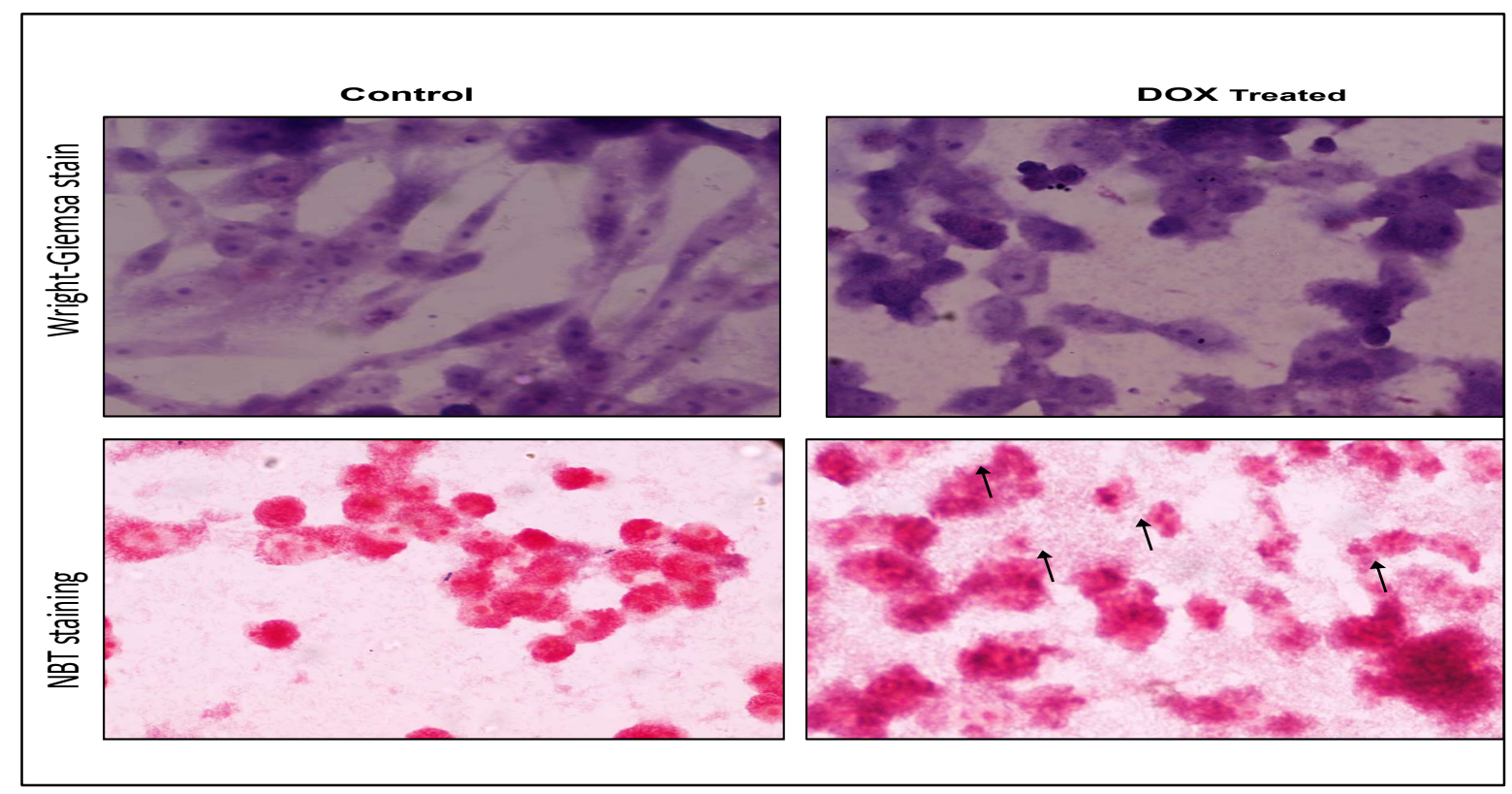

Fig.-2: Change in Cell Morphology and ROS Generation: (A). Change in the morphology of H9c2 cells treated with $5 \mu \mathrm{M}$ DOX concentrations for $24 \mathrm{hr}$ as compared to the control group. (B). Cells stained with NBT to detect purple formazan crystals.

ROS in DOX-treated cardiomyocytes was significantly increased so our next objective was to see its source, inhibitors of classical ROS sources such as NADPH oxidase, Myeloperoxidase, mitochondria and general ROS scavenger (NAC) were used to treat the cardiomyocytes along with DOX. Among the ROS scavengers, DPI, SHX, FCCP and NAC reduced the ROS level by $76 \%, 18 \%, 9 \%$ and $42 \%$ respectively when compared with only DOX treated cells (Fig.-4) confirming that all the ROS scavengers were able to reduce the ROS but DPI and NAC significantly reduced the ROS level. In the previous study, DPI showed a significant ROS scavenging property in DOX influenced environment while the other inhibitor has little role against DOX influenced Cardiotoxicty ${ }^{23}$ whereas, the resulting outcome of NAC also correlates with previous studies where the effect of NAC was countable against the DOX-induced hepatotoxicity. ${ }^{24}$ It implicates that DOX creates an oxidative imbalance either by hindering the activity of antioxidant enzymes, Glutathione reductase or by promoting the role of oxidative stress-inducing NADPH oxidase, which is further supported by other previous reports. ${ }^{25}$

With all these details it was clear that DOX leads to ROS production which further induces oxidative stress. Higher $\mathrm{H}_{2} \mathrm{O}_{2}$ production is detected in DOX-treated cardiomyocytes while among the ROS scavengers, DPI and SHX significantly inhibited the NADPH oxidase and myeloperoxidase (MPO) respectively which prevent the DOX-induced release of $\mathrm{H}_{2} \mathrm{O}_{2}$. NAC maintained the glutathione level as well as increases the activity of manganese superoxide dismutase (MnSOD) which also helps in preventing the $\mathrm{H}_{2} \mathrm{O}_{2}$ production.

Also, DOX is reported with a strong impact on mitochondria through disturbing the respiratory chain complex and process, mitochondrial potential and mitochondrial homeostasis ${ }^{26}$. To confirm the role of mitochondrial ROS, the levels of $\mathrm{H}_{2} \mathrm{O}_{2}$ via an Amplex red assay were evaluated in mitochondria where $17 \%$ more mitochondrial $\mathrm{H}_{2} \mathrm{O}_{2}$ was produced in DOX-treated cardiomyocytes as compared to the untreated ones. DPI, SHX, FCCP and NAC reduced the DOX-induced mitochondrial $\mathrm{H}_{2} \mathrm{O}_{2}$ by $47 \%$, $37 \%, 72 \%$ and $64 \%$ respectively (Fig. -5.1 ). 
RASĀYAN J. Chem.

Vol. 13 | No. 2 |1042 - 1053| April - June | 2020

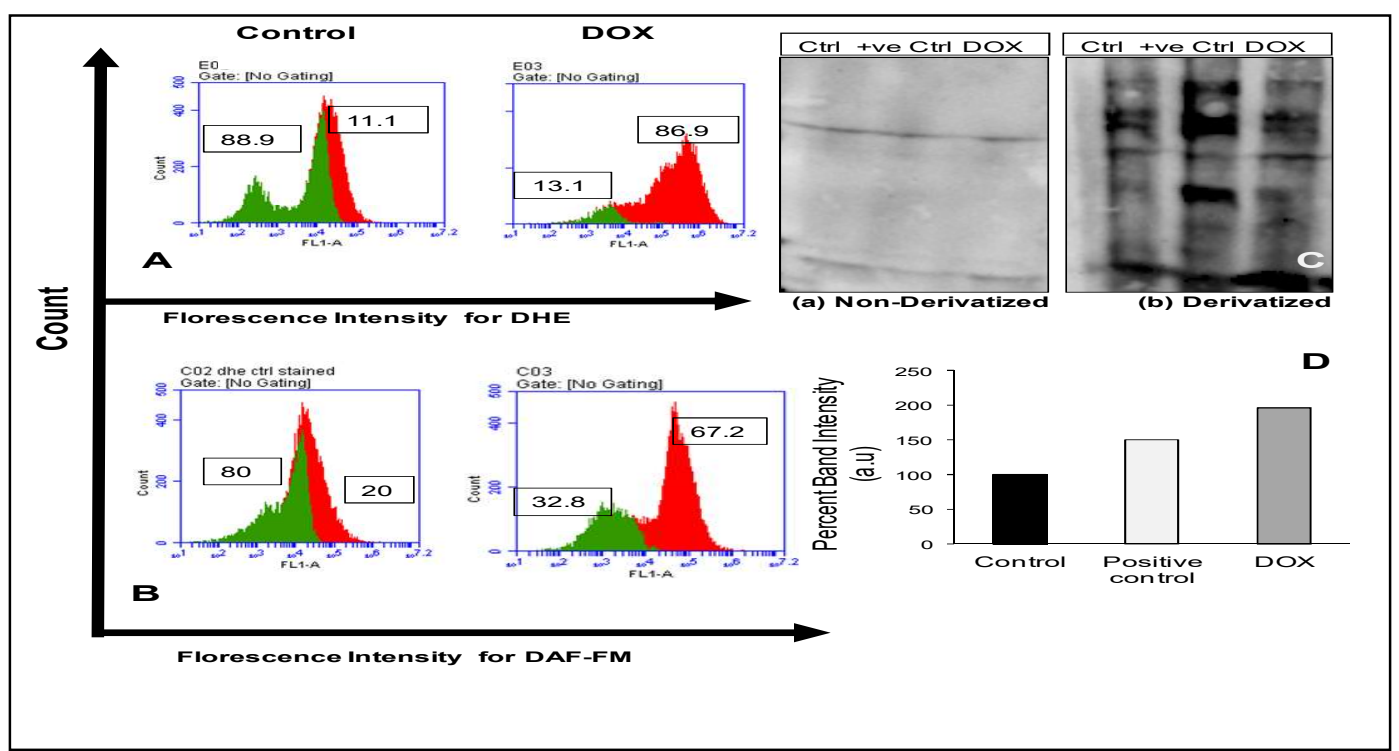

Fig.-3: ROS and Oxidative stress in DOX-treated cardiomyocytes. (A) Flow-cytometry was performed to assess the ROS level using DHE and (B) RNS level using DAF-FM probes, (C) Western blot of Protein Carbonyls: Blot showing protein oxidation in $\mathrm{H} 9 \mathrm{c} 2$ cells treated with $5 \mu \mathrm{M}$ DOX concentration, where figure (a) represents the Non-derivatized samples and figure (b) shows the derivatized samples. (D) Densitometric analysis of Western blot of derivatized protein carbonyl assay. $\mathrm{H}_{2} \mathrm{O}_{2}$ is taken as a positive control.

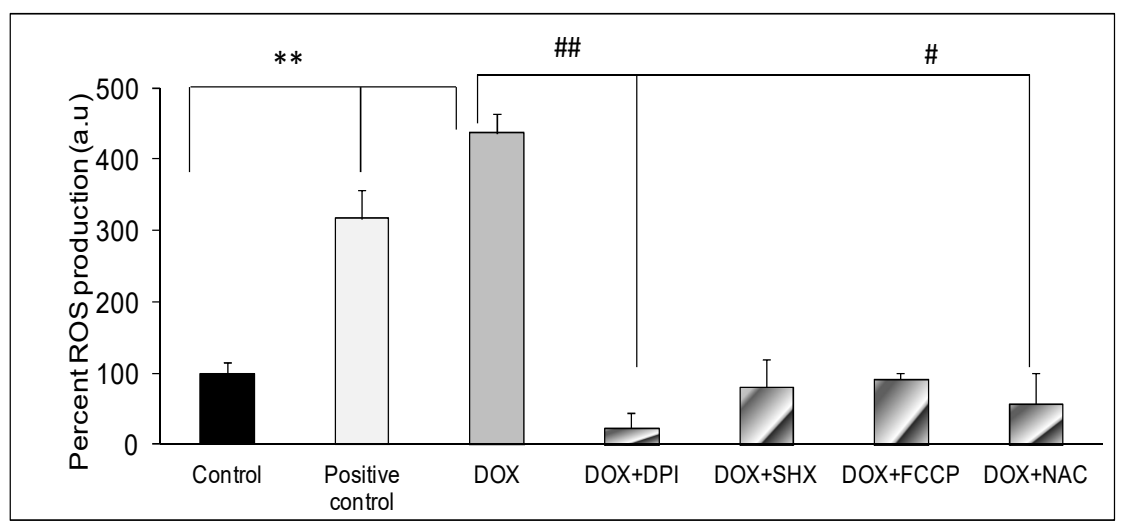

Fig.-4: ROS Production and Inhibition in the DOX treated H9c2 Cells: The percent ROS level detected NBT in H9c2 cells treated with DOX concentration $(5 \mu \mathrm{M})$ for $24 \mathrm{hr}$ and cells were also treated with inhibitors DPI, SHX, FCCP and ROC scavenger NAC. The results are expressed as percent ROS level in arbitrary units [a.u]. Student $t$ test was performed to evaluate the significance of the results, the data was considered as statistically significant at ${ }^{*} \mathrm{p} \leq 0.05$ and ${ }^{* *} \mathrm{p} \leq 0.005$ when DOX-treated cells were compared with untreated controls and ${ }^{\#} \mathrm{p} \leq 0.05$ and ${ }^{\# \#} \mathrm{p} \leq 0.005$ when cells treated with various ROS scavengers along with DOX were compared with only DOX-treated cells. The results are expressed as mean \pm standard deviation $(n=3)$.

FCCP, a mitochondrial ROS scavenger, also implicated that mitochondria also have an influential contribution in the DOX-induced disturbed redox status. Similarly, MPO activity was significantly increased in DOX-treated $\mathrm{H} 9 \mathrm{c} 2$, as a marker of oxidative stress of rats compared to control values. In the previous studies, it is cited that the elevated level of cardiac MPO is correlated with cardiac diseases. ${ }^{27}$ SHX targeting the myeloperoxidase also had some impact on mitochondrial ROS. In support of our data, Hosseini and the group have reported similar outcomes in cancer cells. ${ }^{28}$ NADPH oxidase also regulate the level of mitochondrial ROS. In monocytes/ macrophages, DPI significantly decreases the production of superoxide by mitochondrial respiration through inhibiting the activity of NADPH oxidase. ${ }^{29}$ 
RASĀYAN J. Chem.

Vol. 13 | No. 2 |1042 - 1053| April - June | 2020

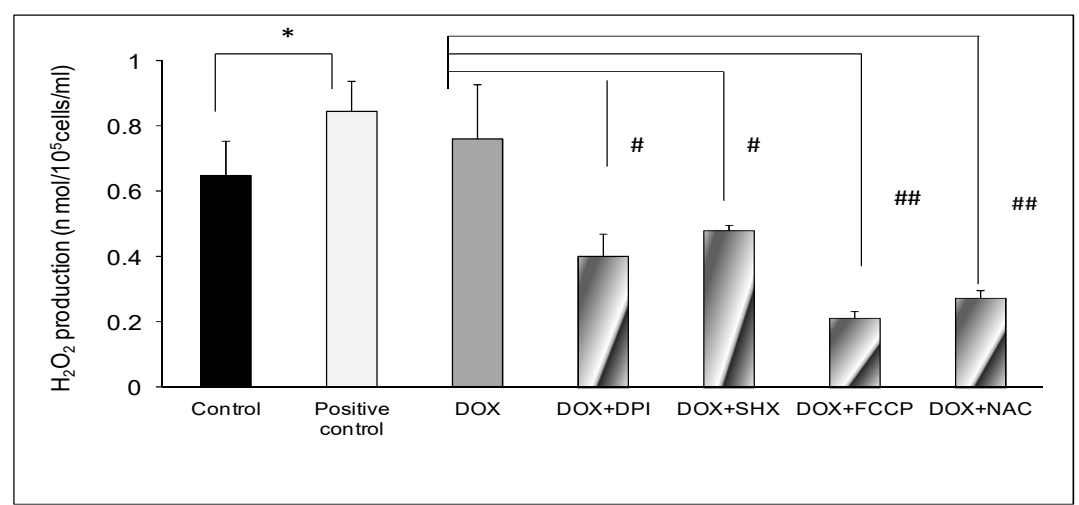

Fig.-5.1: Mitochondrial ROS Production and Inhibition in the DOX treated H9c2 Cells: The percent ROS level detected using Amplex red in mitochondria of H9c2 cells treated with DOX concentration ( $5 \mu \mathrm{M})$ for $24 \mathrm{hr}$ and cells were also treated with inhibitors DPI, SHX, FCCP and ROC scavenger NAC. The results are expressed as percent ROS level in arbitrary units [a.u]. Student $t$ - test was performed to evaluate the significance of the results, the data was considered as statistically significant at ${ }^{*} \mathrm{p} \leq 0.05$ and ${ }^{* *} \mathrm{p} \leq 0.005$ when DOX-treated cells were compared with untreated controls and ${ }^{\#} \mathrm{p} \leq 0.05$ and ${ }^{\#} \mathrm{p} \leq 0.005$ when cells treated with various ROS scavengers along with DOX

were compared with only DOX-treated cells. The results are expressed as mean \pm standard deviation $(n=3)$.

Fluorescent probes MitoSOX (mitochondrial ROS, green fluorescence) and MitoTracker (mitochondria marker, red fluorescence) were used to see the ROS levels in the mitochondrial matrix. Increased green color fluorescence in the overlay image of positive control $\left(\mathrm{H}_{2} \mathrm{O}_{2}\right.$ treated) indicated the increase in ROS production as compared to the control group. In the overlay images of DOX-treated cells, the high intensity of green fluorescence was observed indicating an extra-mitochondrial source of ROS production. Among the ROS inhibitors; DPI, SHX and NAC have reduced the mitochondrial ROS produced by DOX which is visible in the overlay images where the green color intensity of MitoSOX has been reduced as compared to the DOX (Fig.-5.2).

The mitochondrial membrane potential is an important parameter of mitochondrial function and mitochondria health, which can be detected using a fluorescent probe JC-1. In healthy cells with high membrane potential JC-1 forms complex known as J-aggregates with intense red fluorescence while in unhealthy or apoptotic cells with low membrane potential, JC-1 remains in the monomeric form which shows only green fluorescence.

The fluorometric analysis showed that the JC-1 ratio (green monomer / red aggregates) was increased in DOX-treated cardiomyocytes as compared to untreated cells suggesting the low membrane potential of DOX-treated cardiomyocytes as compared to untreated control cardiomyocytes. Among the inhibitors of ROS DPI, SHX and FCCP played a pivotal role as ROS scavengers by reducing the green fluorescence $53 \%, 41 \%$ and $61 \%$ respectively when compared with only DOX-treated cells thus preserved the J-red aggregates as well as the JC-1 ratio when compared to the control level (Fig.-5.3). Thus, these results demonstrate that ROS produced in DOX-treated cardiomyocytes alter the mitochondrial membrane potential but when the other sources of ROS were inhibited the damage to mitochondria was reduced.

DOX decreased the mitochondrial membrane potential of the cardiomyocytes which makes the membrane leaky and allows the release of cytochrome $\mathrm{c}$ from the intermembrane space of mitochondria and initiates the apoptosis cascade. ${ }^{31}$ Western blotting was done to check the release of cytochrome c from the mitochondria of H9c2 cells in the cell lysates. Western blotting and densitometric analysis showed 6 folds more cytochrome c levels than control cells thus confirming the mitochondria damage which leads to the release of cytochrome c. Among the inhibitors, DPI and NAC played an important role in preventing cell death through the release of cytochrome $\mathrm{c}$ by reducing the damage done by DOX up to 5 fold and one fold respectively (Fig.-5.4).The release of cytochrome c accounts for the initiation of apoptosis which was determined with the help of Annexin V-FITC assay. Cells at the early apoptosis stage are Annexin V positive (green fluorescence) and cells at late apoptosis are positive for both Annexin $\mathrm{V}$ and PI (red fluorescence). Maximum cells have undergone late apoptosis in DOX-treated cardiomyocytes that are visible in the overlay images of DOX-treated cells as compared to the control 
RASĀYAN J. Chem.

Vol. 13 | No. 2 |1042 - 1053| April - June | 2020

cells. Among the inhibitors, SHX, NAC and FCCP have played an important role as an antioxidant and prevent apoptosis by DOX ${ }^{32-33}$ (Fig.-6).

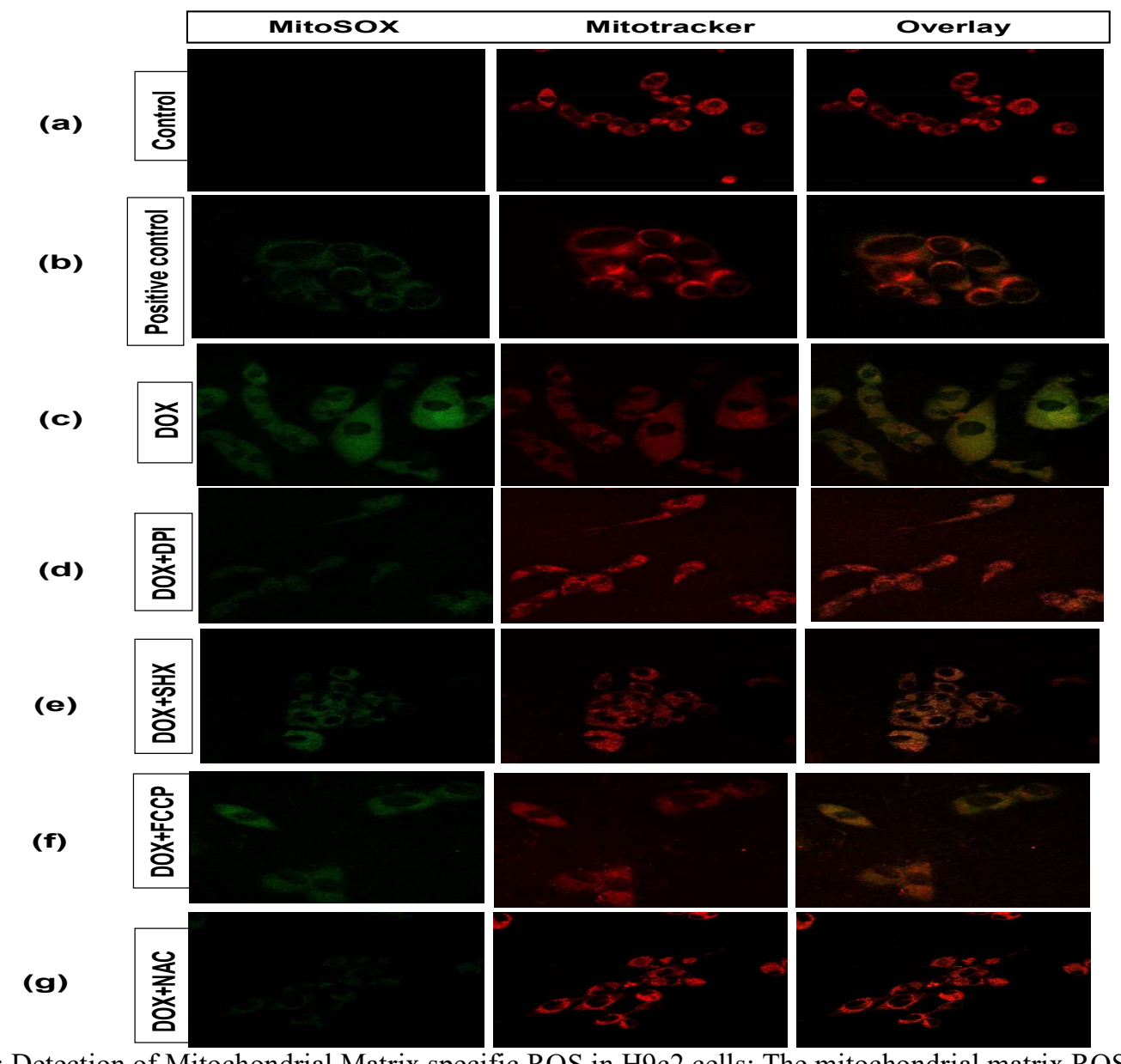

Fig.-5.2: Detection of Mitochondrial Matrix specific ROS in H9c2 cells: The mitochondrial matrix ROS was detected using MitoSOX and MitoTracker in H9c2 cardiomyocytes after various treatments. Where (a) control (b) positive control $\left(\mathrm{H}_{2} \mathrm{O}_{2}\right)(\mathrm{c})$ DOX (d) DOX+DPI (e) DOX+SHX (f) DOX+FCCP (g) DOX+NAC.

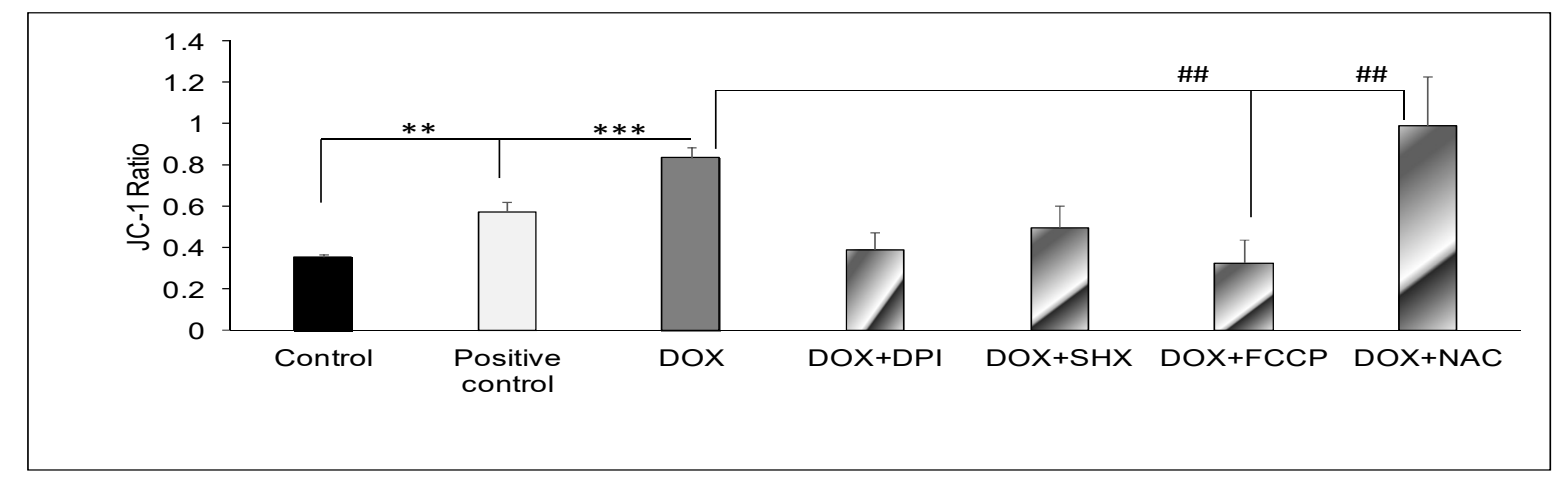

Fig.-5.3: Membrane Potential of H9c2 Cells treated with DOX and ROS Inhibitors: Change in the membrane potential of $\mathrm{H} 9 \mathrm{c} 2$ cells treated with $5 \mu \mathrm{M}$ DOX in the presence of various ROS scavengers was observed. $\mathrm{H}_{2} \mathrm{O}_{2}$ is taken as a positive control. The results were expressed as the JC-1 ratio (green monomer / red aggregates). The results were expressed as the mean \pm standard deviation $(\mathrm{n}=3)$. Student $t$ - test was performed to evaluate the significance of the results, the data was considered as statistically significant at ${ }^{*} \mathrm{p} \leq 0.01$ and ${ }^{* *} \mathrm{p} \leq 0.001$ when DOXtreated cells were compared with untreated controls and ${ }^{* *} \mathrm{p} \leq 0.05$ and ${ }^{\# \#} \mathrm{p} \leq 0.005$ when cells treated with DOX along with various ROS scavengers were compared with only DOX-treated cells. 
RASĀYAN J. Chem.

Vol. 13 | No. 2 |1042 - 1053| April - June | 2020

A

\begin{tabular}{|cllllll}
\hline CTRL Positive ctrl DOX DOX+DPI DOX+SHX DOX+FCCP & DOX+NAC \\
\hline
\end{tabular}

B

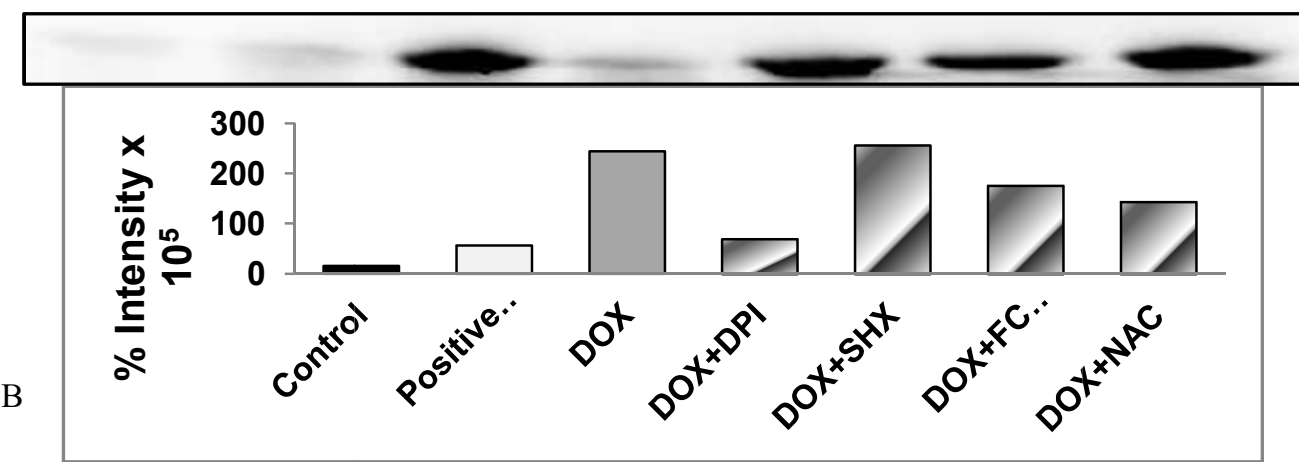

Fig.-5.4: Western blot for Cytochrome $\mathrm{C}$ release: The mitochondrial damage through the release of cytochrome $\mathrm{c}$ in cardiomyocytes treated with $5 \mu \mathrm{M}$ DOX concentrations was seen. $\mathrm{H}_{2} \mathrm{O}_{2}$ is taken as a positive control. (A)

Western Blotting for cytochrome c. (B) Densitometric analysis of Western blot for cytochrome c.

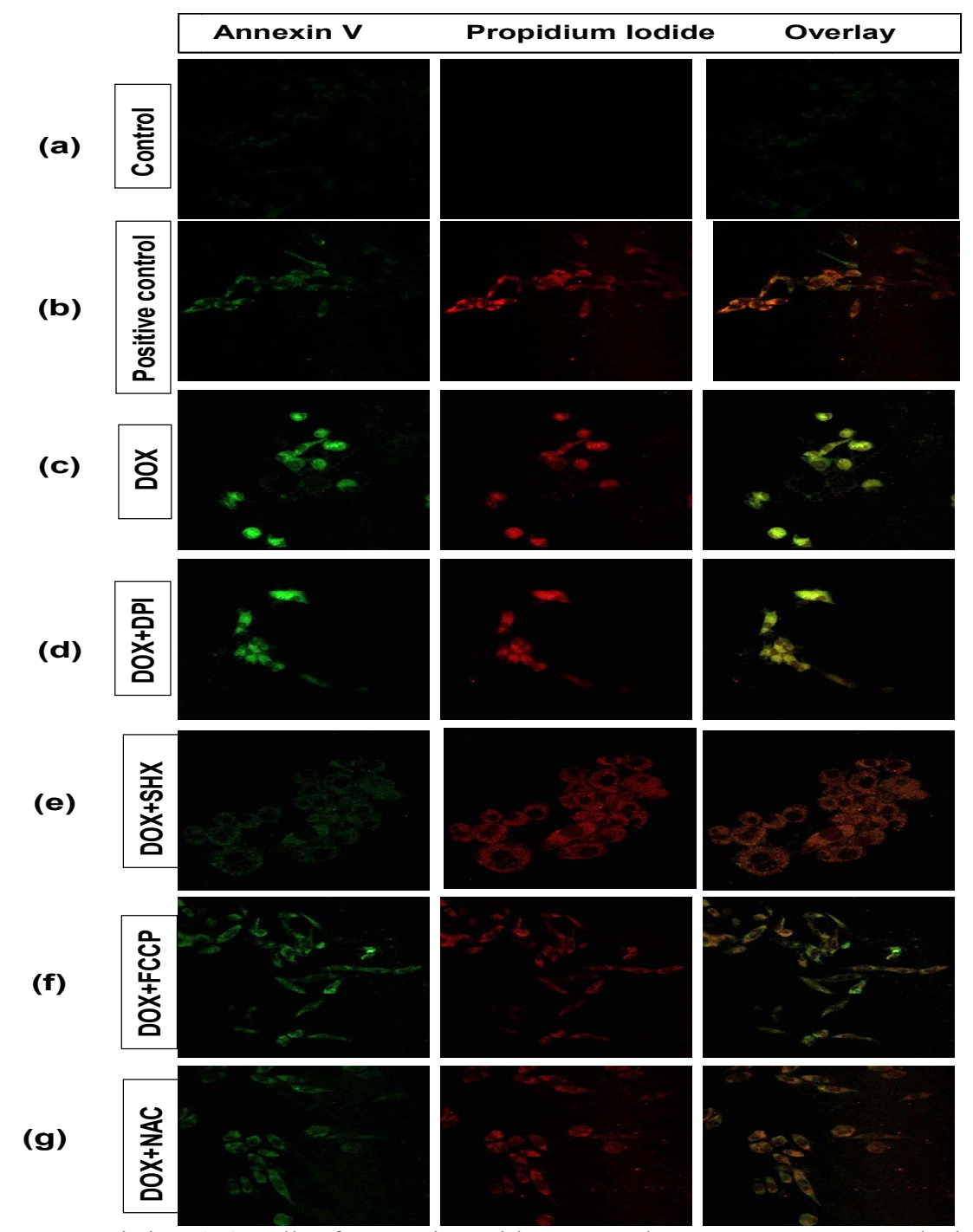

Fig.-6: Intrinsic Apoptosis in H9c2 Cells after treating with DOX and ROS scavengers: Analysis of intrinsic apoptosis with the help of Annexin and PI in DOX-treated cardiomyocytes. Where (a) control, (b) positive control $\left(\mathrm{H}_{2} \mathrm{O}_{2}\right)$, (c) DOX, (d) DOX+DPI, (e) DOX+SHX, (f) DOX+FCCP, (g) DOX+NAC. 
In the previous studies, it has been reported that DOX enhances oxidative stress by increasing the level of ROS through various pathways which leads to mitochondrial dysfunction. DOX tends to accumulate in the mitochondria of cardiac cells which affect the functioning of topoisomerase-II and cause damage to the mitochondrial DNA which ultimately affects the electron transport chain (ETC). DOX is also known to disturb the $\mathrm{Ca}^{2+}$ balance of the mitochondria which stimulates the release of cytochrome $\mathrm{c}$ and initiates the apoptosis. ${ }^{34}$ DOX produces ROS by uncoupling of NOS, through NADPH oxidase and via forming Fe-DOX complex. ${ }^{35}$ The ROS produced by DOX leads to DNA damage, lipid peroxidation as well as apoptosis. ${ }^{36-37}$ This immense damage to cardiac mitochondria is the root cause of cardiotoxicity.

In summary, DOX kills the tumor cells by intercalating into the nuclear DNA, inhibits the function of topoisomerase-II, generates ROS, and induces apoptosis; but the problem arises when it starts doing same in the normal cells. The present study has identified the mechanism of DOX-induced ROS production which leads to mitochondrial dysfunction and hence cardiotoxicity. H9c2 rat cardiomyocytes were treated with $5 \mu \mathrm{M}$ DOX concentration and an increase in ROS was observed in the mitochondria which lead to mitochondrial dysfunction and play a vital role in cardiotoxicity. Inhibiting various classical ROS sources such as NADPH oxidase, myeloperoxidase, mitochondria and general ROS scavenger (NAC) suggested that ROS via NOX and MPO during DOX-induced toxicity plays a crucial role in cardiomyocytes. This study will provide some directions for the development of adjunct therapies to control oxidative disarray happening during chemotherapy resulting in heart failure and other related complications.

\section{CONCLUSION}

Based on our findings and previous reports it is suggested that the use of chemotherapeutic drugs should be followed closely for symptoms and/or signs of cardiac damage and dysfunction. Our findings highlight the importance of conducting more detailed preclinical studies to determine the mechanism of cardiotoxicity via ROS mediated pathways specifically mitochondrial ROS and NADPH oxidase or MPO depended ROS should be explored in other chemotherapeutic drugs and to develop strategies to prevent cardiac dysfunction and more promising cancer therapeutics.

\section{ACKNOWLEDGMENT}

Shishir Upadhayay and Nidhi Sharma have contributed equally to the present manuscript. Shishir acknowledges the ICMR-JRF/SRF, Nidhi Sharma acknowledges the student fellowship from the Central University of Punjab, Bathinda (CUPB). The authors also acknowledge the technical facilities provided by the Central Instrumentation Facility, CUPB. This work is supported by the DST fast track grant (SB/4S/LS-107/2013) to Dr. Dhiman.

\section{REFERENCES}

1. M. Valko, D. Leibfritz, J. Moncol, M.T. Cronin, M. Mazur, and J. Telser, The International Journal of Biochemistry and Cell Biology 39, 44(2007).

2. D. Montaigne, C. Hurt, and R. Neviere, Biochemistry Research International 2012, 1(2012).

3. M. Dhiman, S. Thakur, S. Upadhyay, A. Kaur, and A. K. Mantha, (2015), Oxidative Stress and Inflammation in Cardiovascular Diseases: Two Sides of the Same Coin, Free Radicals in Human Health and Disease, Springer, India, pp.259-278

4. X. Ma, and Y. Herbert, The Yale Journal of Biology and Medicine, 79(3-4), 85 (2006).

5. A. M. Rahman, S. W. Yusuf, and M. S. Ewer, International Journal of Nanomedicine, 2(4), 567(2007).

6. G. Minotti, P. Menna, E. Salvatorelli, G. Cairo, and L. Gianni, Pharmacological Reviews, 56(2), 185(2004).

7. Y. Alfaro, G. Delgado, A. Cárabez, B. Anguiano, and C. Aceves, Molecular Cancer, 12(1), 1 (2013).

8. M. Dhiman, M.P. Zago, S. Nunez, A. Amoroso, H. Rementeria, P. Dousset, F. Burgos, and N.J. Garg, PLoS One 7(1), e28449 (2012), DOI:10.1371/journal.pone.0028449. Epub 2012 Jan 4.

9. H. S. Choi, J.W. Kim, Y.N. Cha, and C. Kim, Journal of Immunoassay and Immunochemistry, 27(1), 31(2006).

10. S. Upadhyay, S. Vaish, and M. Dhiman, Molecular and Cellular Biochemistry, 450(1-2), 135(2019). 
RASĀYAN J. Chem.

Vol. 13 | No. 2 |1042 - 1053| April - June | 2020

11. I. Gill, S. Kaur, N. Kaur, M. Dhiman, and A.K. Mantha, Journal of Alzheimer's Disease, 60(s1), S25(2017).

12. V. Rhein, M. Giese, G. Baysang, F. Meier, S. Rao, K.L. Schulz, and A. Eckert, PLoS One 5(8), e12359 (2010), DOI:10.1371/journal.pone.0012359.

13. N. Kaur, M. Dhiman, J.R. PerezPolo, and A.K. Mantha, Journal of Neuroscience Research 93(6), 938(2015).

14. K.B. Gupta, S. Upadhyay, R.G. Saini, A.K. Mantha, and M. Dhiman, Journal of Cereal Science, 81, 91(2018).

15. S. Thakur, M. Dhiman and A.K. Mantha, Molecular and Cellular Biochemistry, 441(1-2), 201(2018).

16. S. Gupta, V. Bhatia, J-J Wen, Y. Wu, M.H. Huang, and N.J. Garg, Free Radical Biology and Medicine 47(10), 1414(2009).

17. T. Sugawara, M. Fujimura, Y. Morita-Fujimura, M. Kawase, and P.H. Chan, Journal of Neuroscience, 19, RC39-1 (1999).

18. S. Kumar, V. Kain, and S.L. Sitasawad, Biochimica et Biophysica Acta (BBA)-General Subjects, 1820(7), 907(2012).

19. J. P. Sánchez-Villamil, V. D'Annunzio, P. Finocchietto, S. Holod, I. Rebagliati, H. Pérez and M.C. Carreras, The International Journal of Biochemistry and Cell Biology, 81, 323(2016).

20. D.I. Brown, and K. K. Griendling, Circulation Research, 116(3), 531(2015).

21. A. Daugherty, J. L. Dunn, D. L. Rateri and J.W. Heinecke, Myeloperoxidase, The Journal of Clinical Investigation, 94(1), 437(1994).

22. B. Aryal, J. Jeong, and V. A. Rao, Proceedings of the National Academy of Sciences, 111(5), 2011(2014).

23. D. McLaughlin, Y. Zhao, K. M. O'neill,K. S. Edgar,P.D. Dunne, A. M. Kearney, and B. J. McDermott, British Journal of Pharmacology, 174(21), 3677(2017).

24. M. C. Koçkar, M. Nazıroğlu, O. Çelik, H. T. Tola,D. Bayram, and A. Koyu, Cell Biochemistry and Function, 28(8), 673(2010).

25. J. D. Lambeth, J. D. NOX,Nature Reviews Immunology, 4(3), 181 (2004).

26. S. Gorini, A. De Angelis, L. Berrino, N. Malara, G. Rosano, and E. Ferraro, Oxidative Medicine and Cellular Longevity, (2018), DOI: 10.1155/2019/9601435.

27. A. A. Hamza, M. M. Ahmed, H. M. Elwey, and A. Amin, PLoS One, 11(11), e0167049(2016), DOI: 10.1371/journal.pone.0167049. eCollection 2016.

28. M. Hosseini, H. R. Rezvani, N. Aroua, C. Bosc, T. Farge, E. Saland, and M. Sabatier, Cancer Research, 79(20), 5191(2019).

29. Y. Li, and M. A. Trush, Biochemical and Biophysical Research Communications, 253(2), 295(1998).

30. M. Dhiman and N. J. Garg, PLoS Pathogens, 4,10(12),e1004516(2014), DOI: 10.1371/journal.ppat.1004516.

31. J. Cai, J. Yang, and D. Jones, Biochimica et Biophysica Acta (BBA)-Bioenergetics 1366(1), 139(1998).

32. M. Wasilewski, and L. Wojtczak, FEBS letters, 579(21), 4724(2005).

33. T. Kirchner, S. Möller, M. Klinger, W. Solbach, T. Laskay, and M. Behnen, Mediators of Inflammation 2012, 1(2012).

34. M.A. Mitry, and J.G. Edwards, IJC Heart and Vasculature 10, 17(2016).

35. Y. Octavia, C.G. Tocchetti, K.L. Gabrielson, S. Janssens, H.J. Crijns, and A. L. Moens, Journal of Molecular and Cellular Cardiology 52, 1213(2012).

36. M. Zeviani, and C. Antozzi, Molecular Human Reproduction, 3, 133(1997).

37. M. Ott, V. Gogvadze, S. Orrenius, and B. Zhivotovsky, Apoptosis 12, 913(2007).

[RJC-5603/2019] 